\title{
Surface modes and photonic modes in Casimir calculations for a compact cylinder
}

\author{
V V Nesterenko \\ Bogoliubov Laboratory of Theoretical Physics, Joint Institute for Nuclear Research, \\ Dubna 141 980, Russia \\ E-mail: nestr@theor.jinr.ru
}

\begin{abstract}
A rigorous formulation of the problem of calculating the electromagnetic vacuum energy of an infinite dielectric cylinder is discussed. It is shown that the physically relevant spectrum of electromagnetic excitations includes the surface modes and photonic modes. The mathematical procedure of summing over this spectrum is proposed, and the transition to imaginary frequencies is accomplished. As a result, it is justified the imaginary-frequency representation for the vacuum energy which has been used in previous Casimir studies for this configuration.

PACS numbers: 11.10.Gh; 42.50.Pq; 03.70.+k; 03.65.Sq, 11.30.Ly
\end{abstract}

\section{Introduction}

The notion of the elementary excitation spectrum is of paramount importance in all condensed matter physics [1, 2]. The excitations of different type result, as a rule, in different physical consequences. Therefore it is of a certain interest for theoretical and experimental investigations of the Casimir effect to answer the question: the electromagnetic oscillations of what type are considered in the problem at hand [3, 4, 5, 6, 7, 8. Having elucidated this point one can hope to link, in a transparent way, the Casimir force with actual physical properties of the material boundaries. However it is not easy to answer this question even when the Casimir force is calculated by making use of the familiar Lifshitz formula [9, 10, 11]. A rather complicated derivation of this formula [12] in the original papers initiated its obtaining anew by making use mainly of the mode summation method [12, 13, 14, 15, 16, 17, 18, 19].

The Casimir calculations for nonflat boundaries turned out to be much more involved in comparison with those for planes [20]. Especially complicated calculations have been done for a circular cylinder [21, 22, 223, 24, 25, 26, 27, 28, 29, 30, 31, 32, 33, 34, 35, 36, 37, 38. It was also unclear electromagnetic excitations of what kind have been taken into account in these studies [39].

The present work seeks to present a consistent derivation of the formula for the vacuum energy of electromagnetic field connected with a material cylinder by 
summing explicitly the contributions to this energy given by different branches of the electromagnetic spectrum in this problem.

The layout of the paper is as follows. In Sec. 2 the spectral problem generated by the Maxwell equations for a compact infinite cylinder is formulated rigorously and the physically relevant spectrum of electromagnetic excitations for this configuration is determined. It is shown that this spectrum includes surface modes (bound states) and photonic modes. In Sec. 3 the summation over this spectrum is accomplished by making use of the spectral density when accounting of the photonic (continues) branch of the spectrum. In Conclusion (Sec. 4) the meaning of obtained results are discussed briefly.

\section{Physical spectrum of electromagnetic excitations for a cylinder}

In the source-free case the general solution to Maxwell equations can be represented in terms of two independent Hertz vectors [40]

$$
\begin{aligned}
& \mathbf{E}=\nabla \times \nabla \times \boldsymbol{\Pi}^{\prime}+i \mu \frac{\omega}{c} \nabla \times \boldsymbol{\Pi}^{\prime \prime}, \\
& \mathbf{H}=-i \varepsilon \frac{\omega}{c} \nabla \times \boldsymbol{\Pi}^{\prime}+\nabla \times \nabla \times \boldsymbol{\Pi}^{\prime \prime} .
\end{aligned}
$$

Here $\boldsymbol{\Pi}^{\prime}$ is the electric Hertz vector, $\boldsymbol{\Pi}^{\prime \prime}$ is the magnetic Hertz vector, $c$ is the velocity of light in vacuum, and the Gauss units are used. The Hertz vectors obey the Helmholtz vector equation

$$
\left(\nabla^{2}+k^{2}\right) \Pi=0,
$$

where the wave number $k$ is given by

$$
k^{2}=\varepsilon \mu \frac{\omega^{2}}{c^{2}} .
$$

On the other hand, it is known that the general solution to Maxwell without sources equations can be derived from two scalar functions which may be chosen in deferent ways [41, 42]. For the configuration with cylindrical symmetry the role of these functions can play the axial components of the electric $\left(\boldsymbol{\Pi}^{\prime}\right)$ and magnetic $\left(\boldsymbol{\Pi}^{\prime \prime}\right)$ Hertz vectors. The rest components of $\boldsymbol{\Pi}^{\prime}$ and $\boldsymbol{\Pi}^{\prime \prime}$ are zero in this case. As a result, the Helmholtz vector equation (3) reduces to the scalar Helmholtz equations for $\Pi_{z}^{\prime} \equiv \Pi^{\prime}$ and $\Pi_{z}^{\prime \prime} \equiv \Pi^{\prime \prime}$

$$
\left(\Delta+\varepsilon \mu \frac{\omega^{2}}{c^{2}}\right) \Pi=0, \quad \Pi=\Pi^{\prime}, \Pi^{\prime \prime}
$$

with the following general solutions

$$
\begin{aligned}
\Pi^{\prime} & =\sum_{n=0, \pm 1, \pm 2, \ldots} a_{n} f_{n}^{\mathrm{TM}}(r) e^{i h z+i n \theta}, \\
\Pi^{\prime \prime} & =\sum_{n=0, \pm 1, \pm 2, \ldots} b_{n} f_{n}^{\mathrm{TE}}(r) e^{i h z+i n \theta} .
\end{aligned}
$$

The cylindrical coordinates $(r, \theta, z)$ are used and the $z$ axis coincides with the axis of a circular infinite cylinder of radius $a$. The medium inside the cylinder has the permittivity $\varepsilon_{1}$ and permeability $\mu_{1}$. These quantities outside the cylinder acquire the values $\varepsilon_{2}$ and 
$\mu_{2}$, respectively. We assume for definiteness that $\varepsilon_{1} \mu_{1}>\varepsilon_{2} \mu_{2}$. The wave vector along the $z$ axis is denoted by $h$. The amplitudes $a_{n}$ and $b_{n}$ for the solutions inside the cylinder will be denoted by $a_{n}^{i}$ and $b_{n}^{i}$, respectively, and in the same way for solutions outside the cylinder we introduce the amplitudes $a_{n}^{e}$ and $b_{n}^{e}$.

The functions $f_{n}^{\mathrm{TE}}(r)$ and $f_{n}^{\mathrm{TM}}(r)$ in the general solutions (6) and (7) obey the radial wave equation

$\frac{d^{2} f_{n}}{d r^{2}}+\frac{1}{r} \frac{d f_{n}}{d r}+\left(k^{2}-h^{2}-\frac{n^{2}}{r^{2}}\right) f_{n}=0, \quad f_{n}(r)=f_{n}^{\mathrm{TE}}(r), f_{n}^{\mathrm{TM}}(r)$.

Inside the cylinder we put

$$
f_{n}(r)=J_{n}\left(\lambda_{1} r\right), \quad n=0,1, \ldots, \quad 0<r<a,
$$

where $J_{n}(z)$ is the Bessel function, $\lambda_{1}=\sqrt{k_{1}^{2}-h^{2}}, k_{1}^{2}=\omega^{2} / c_{1}^{2}=\varepsilon_{1} \mu_{1} \omega^{2} / c^{2}$. Outside the cylinder we consider first 'outgoing' waves

$$
f_{n}(r)=H_{n}^{(1)}\left(\lambda_{2} r\right), \quad n=0,1, \ldots, \quad r>a,
$$

where $H^{(1)}(z)$ is the Hankel function of the first kind, $\lambda_{2}=\sqrt{k_{2}^{2}-h^{2}}, k_{2}^{2}=\omega^{2} / c_{2}^{2}=$ $\varepsilon_{2} \mu_{2} \omega^{2} / c^{2}$.

In the radial solutions (9) and (10) the sign of $\lambda_{s}^{2}=k_{s}^{2}-h^{2}, s=1,2$ is not fixed yet. Thus, in our consideration the solutions

$$
f_{n}(r)=I_{n}\left(\bar{\lambda}_{1} r\right) \quad \text { for } \quad r<a
$$

and

$$
f_{n}(r)=K_{n}\left(\bar{\lambda}_{2} r\right) \quad \text { for } \quad r>a
$$

are also admissible. Here $\bar{\lambda}_{s}^{2}=h^{2}-k_{s}^{2}, \quad s=1,2, I_{n}(z)=\mathrm{i}^{-n} J_{n}(\mathrm{i} z)$ and $K_{n}(z)=$ $\mathrm{i}^{n+1} \frac{\pi}{2} H_{n}(\mathrm{i} z)$ are the modified Bessel functions [43].

On the cylinder surface the matching conditions should be satisfied. These conditions require the continuity of tangential components of fields $\mathbf{E}$ and $\mathbf{H}$ when crossing cylinder surface

$$
\operatorname{discont}\left(\mathbf{E}_{\|}\right)=0, \quad \operatorname{discont}\left(\mathbf{H}_{\|}\right)=0 .
$$

The matching conditions give rise to the frequency equation determining admissible values of the spectral parameter $\omega$ in the boundary value problem under consideration:

$$
\begin{aligned}
\frac{\omega^{2} a^{4}}{c^{2}}\left(\varepsilon_{1} \lambda_{2} \frac{J_{n}^{\prime}}{J_{n}}\right. & \left.-\varepsilon_{2} \lambda_{1} \frac{H_{n}^{\prime}}{H_{n}}\right)\left(\mu_{1} \lambda_{2} \frac{J_{n}^{\prime}}{J_{n}}-\mu_{2} \lambda_{1} \frac{H_{n}^{\prime}}{H_{n}}\right) \\
& -\frac{n^{2} h^{2} a^{2}}{\lambda_{1}^{2} \lambda_{2}^{2}}\left[\frac{\omega^{2}}{c^{2}}\left(\varepsilon_{1} \mu_{1}-\varepsilon_{2} \mu_{2}\right)\right]^{2}=0, \quad n=0,1,2, \ldots .
\end{aligned}
$$

In this equation

$$
J_{n} \equiv J_{n}\left(\lambda_{1} a\right), \quad H_{n} \equiv H_{n}^{(1)}\left(\lambda_{2} a\right), \quad \lambda_{s}=+\sqrt{\omega^{2} / c_{s}^{2}-h^{2}}, \quad s=1,2,
$$

the prime on the functions $J_{n}$ and $H_{n}$ means differentiation with respect to their arguments, $c_{1}$ and $c_{2}$ are the velocities of light inside and outside the cylinder, respectively, $c_{2}>c_{1}$. 
The roots of (14) are important in radio-engineering when developing the radio dielectric waveguides [44, 45, 46, 47, 48, 49, 50] and in fiber optics (optical waveguides [51, 52]). The results of investigation of the frequency equation (14) determining the spectrum in the problem at hand can be summarized in the following way. All the real roots of this equation lie in the interval

$$
c_{1} h<\omega<c_{2} h .
$$

These roots make up two discrete sequences. In the interval (16) the frequency equation (14) can be rewritten in the form

$$
\begin{aligned}
\frac{\omega^{2} a^{4}}{c^{2}}\left(\varepsilon_{1} \bar{\lambda}_{2} \frac{J_{n}^{\prime}}{J_{n}}\right. & \left.+\varepsilon_{2} \lambda_{1} \frac{K_{n}^{\prime}}{K_{n}}\right)\left(\mu_{1} \bar{\lambda}_{2} \frac{J_{n}^{\prime}}{J_{n}}+\mu_{2} \lambda_{1} \frac{K_{n}^{\prime}}{K_{n}}\right) \\
& -\frac{n^{2} h^{2} a^{2}}{\lambda_{1}^{2} \bar{\lambda}_{2}^{2}}\left[\frac{\omega^{2}}{c^{2}}\left(\varepsilon_{1} \mu_{1}-\varepsilon_{2} \mu_{2}\right)\right]^{2}=0, \quad n=0,1,2, \ldots
\end{aligned}
$$

with the notation $K_{n} \equiv K_{n}\left(\bar{\lambda}_{2} a\right)$.

When frequency $\omega$ equals the real roots of equation (17), located in the interval (16), the 'outgoing' waves (10) become the functions decaying in the radial direction (12) (surface or evanescent waves). These eigenmodes describe the propagation of electromagnetic waves along the cylinder (waveguide solutions). The radial functions (11) are not realized in the problem under consideration.

Now we address the complex roots of the frequency equation (14). In the strip of the complex frequency plane

$$
0<\operatorname{Re} \omega<c_{2} h
$$

there are no complex roots of (14) with $\operatorname{Im} \omega \neq 0$.

In the semi-plane

$$
\operatorname{Re} \omega>c_{2} h
$$

for sure there are complex roots of (14) with $\operatorname{Im} \omega \neq 0$. Indeed, in the domain (19) the left-hand side of (14) is a complex function of the complex variable $\omega$. The complex eigenfrequencies of a dielectric cylinder lead to leaky (radiating) modes. It is clear that these modes cannot carry the electromagnetic energy along the cylinder. For us it is important that the modes with complex $\omega$ (quasi-normal modes [39]) do not satisfy standard completeness condition and as a result they cannot be used for quantization of electromagnetic field in the problem at hand.

In order to get rid of the complex eigenfrequencies and consequently to escape leaky or radiating modes we shall consider, outside the cylinder, the scattering states instead of outgoing waves. The scattering solutions to Maxwell equations can be derived from outgoing solutions by making use of the substitutions

$$
\begin{aligned}
& a_{n}^{e} H_{n}^{(1)}\left(\lambda_{2} r\right) \rightarrow a_{n}^{+} H_{n}^{+}\left(\lambda_{2} r\right)+a_{n}^{-} H_{n}^{-}\left(\lambda_{2} r\right) \\
& b_{n}^{e} H_{n}^{(1)}\left(\lambda_{2} r\right) \rightarrow b_{n}^{+} H_{n}^{+}\left(\lambda_{2} r\right)+b_{n}^{-} H_{n}^{-}\left(\lambda_{2} r\right)
\end{aligned}
$$

For simplicity in (20) the notations

$$
H_{n}^{+} \equiv H_{n}^{(1)}, \quad H_{n}^{-} \equiv H_{n}^{(2)}
$$


are introduced.

As a result, for a given $n$ and $h$ we have 6 amplitudes $a_{n}^{i}, b_{n}^{i}, a_{n}^{+}, a_{n}^{-}, b_{n}^{+}, b_{n}^{-}$. The matching conditions at the cylinder surface lead to 4 linear homogenous equations for these amplitudes. Hence no restrictions arise here for the spectral parameter $\omega^{2} / c^{2}$.

Eliminating in these equations the amplitudes $a_{n}^{i}$ and $b_{n}^{i}$ we are left with 2 equations in 4 amplitudes $a_{n}^{ \pm}$and $b_{n}^{ \pm}$

$$
K^{-}\left(\begin{array}{c}
a_{n}^{+} \\
b_{n}^{+}
\end{array}\right)=K^{+}\left(\begin{array}{c}
a_{n}^{-} \\
b_{n}^{-}
\end{array}\right), \quad K^{ \pm}=\mp\left(\begin{array}{cc}
\alpha^{\mp} & \beta^{\mp} \\
\gamma^{\mp} & \alpha^{\mp}
\end{array}\right),
$$

where

$$
\begin{aligned}
& \alpha_{n}^{ \pm}=\frac{n h}{a}\left(-1+\frac{\lambda_{2}^{2}}{\lambda_{1}^{2}}\right) \\
& \beta_{n}^{ \pm}=-\mathrm{i} \frac{\omega}{c}\left(\mu_{2} \lambda_{2} H_{n}^{ \pm^{\prime}}-\mu_{1} \lambda_{1} \frac{J_{n}^{\prime}}{J_{n}} \frac{\lambda_{2}^{2}}{\lambda_{1}^{2}} H_{n}^{ \pm}\right), \\
& \gamma_{n}^{ \pm}=\mathrm{i} \frac{\omega}{c}\left(\varepsilon_{2} \lambda_{2} H_{n}^{ \pm^{\prime}}-\varepsilon_{1} \lambda_{1} \frac{J_{n}^{\prime}}{J_{n}} \frac{\lambda_{2}^{2}}{\lambda_{1}^{2}} H_{n}^{ \pm}\right)
\end{aligned}
$$

The $S$ matrix in this problem

$$
\left(\begin{array}{c}
a_{n}^{+} \\
b_{n}^{+}
\end{array}\right)=S\left(\begin{array}{c}
a_{n}^{-} \\
b_{n}^{-}
\end{array}\right)
$$

obeys obviously the following matrix equation

$$
K^{-} S=K^{+}
$$

and

$$
\operatorname{det} S=\frac{\operatorname{det} K^{+}}{\operatorname{det} K^{-}}
$$

By a direct calculation one can easily show that det $K^{-}$coincides (up to unimportant multiplier) with the left-hand side of the frequency equation (14). Thus this equation can be rewritten in the form

$$
\operatorname{det} K^{-}=0 \text {. }
$$

Surprisingly formulas (26) and (27) for the $S$ matrix were not known in the literature devoted to the electromagnetic scattering by a cylinder.

Summarizing we infer that the spectrum of electromagnetic oscillations in the problem under study consists of discrete values $\omega_{n \alpha}: c_{1} h<\omega_{n \alpha}<c_{2} h$ corresponding to the surface modes and a continuous branch of the spectrum with real positive $\omega$ : $c_{2} h<\omega<\infty$. In mathematical scattering theory [53] it is proved that the bound states and scattering states form together a complete set. 


\section{Summation over the spectrum and transition to imaginary frequencies}

Now we address the calculation of the vacuum energy in the problem at hand proceeding from the standard mode-by-mode summation

$E_{c}=\frac{1}{2} \sum_{\{q\}} \omega_{q}=\int_{-\infty}^{\infty} \frac{\mathrm{d} h}{2 \pi} \sum_{n=0}^{\prime}\left[\sum_{\alpha} \omega_{n \alpha}(h)+\int_{c_{2} h}^{\infty} \omega \Delta \rho_{n}(\omega, h) \mathrm{d} \omega\right]$,

where the prime over the sum sign means that the term with $n=0$ is taken with the weight $\frac{1}{2}$. The first term in square brackets is responsible for the surface waves contribution and the second one describes the contribution of the photonic modes. The latter contribution is represented by making use of the respective spectral shift function $\Delta \rho$ [39]. The rigorous mathematical scattering theory gives the following expression for the spectral density shift

$$
\Delta \rho(k) \equiv \rho(k)-\rho_{0}(k)=\frac{1}{2 \pi i} \frac{d}{d k} \operatorname{tr} \ln S(k)=\frac{1}{2 \pi i} \frac{d}{d k} \ln \operatorname{det} S(k) .
$$

Here $\rho(k)$ is the density of states for a given potential (or for a given boundary conditions in the case of compound media) and $\rho_{0}(k)$ is the spectral density in the respective free spectral problem (for vanishing potential or for homogeneous unbounded space). It is obvious that in the Casimir calculations one has to use just $\Delta \rho(k)$ subtracting at this point the so-called Minkowski space-time contribution to the vacuum energy.

In the case of scalar scattering problem the Jost functions $f(k)$ and $f(-k)$, the scattering matrix $S(k)$, and the phase shift $\delta(k)$ are related by the formula

$$
S(k)=e^{2 i \delta(k)}=\frac{f(k)}{f(-k)} .
$$

Substitution of (30) into (28) gives more familiar formula for spectral density [54]

$$
\Delta \rho(k)=\frac{1}{2 \pi i} \frac{d}{d k} \ln \frac{f(k)}{f(-k)}=\frac{1}{\pi} \frac{d}{d k} \delta(k) .
$$

In the problem under consideration the TE and TM modes do not decouple. Therefore we are dealing here with the matrix $(2 \times 2)$ scattering problem and we must use the spectral density defined by (29).

The contribution of the surface modes in (28) can be represented by the counter intergral

$$
\sum_{\alpha} \omega_{n \alpha}=\frac{1}{2 \pi \mathrm{i}} \oint_{C} \omega \frac{\mathrm{d}}{\mathrm{d} \omega} \ln F_{n}(\omega) \mathrm{d} \omega,
$$

where $F_{n}(\omega)$ is the left hand side of (17). This equation was written for real $\omega$. However, in the counter integral (32) an analytical continuation of this function to the complex frequency plane should be used. It can be done immediately in terms of $\operatorname{det} K^{+}$(lower semi-plane $\omega$ ) and det $K^{-}$(upper semi-plane $\omega$ ). After that we can use for both terms in (28) the counter integral representations with the counters $C_{+}$and $C_{-}$, respectively. The counter $C_{-}$starts at i $\infty$ and goes along the positive imaginary axis to the origin and after that it goes along the positive real semi-axis to infinity. The counter $C_{+}$is 
obtained by refection of $C_{-}$to the upper semi-plane $\omega$. As a result we arrive at the following imaginary frequency representation of the vacuum energy in the problem at hand

$$
E_{c}=\int_{-\infty}^{\infty} \frac{\mathrm{d} h}{2 \pi} \sum_{n=0}^{\infty} \int_{0}^{\infty} y \frac{\mathrm{d}}{\mathrm{d} y} \ln F_{n}(\mathrm{i} y, h) \mathrm{d} y,
$$

where $F_{n}(\omega, h)$ is the left hand side of the frequency equation (14). It is this representation that has been used in the Casimir calculations for a material cylinder.

\section{Conclusion}

We have shown that in the case of a material cylinder there are two types of electromagnetic excitations which are physically relevant: i) surface modes and ii) photonic modes. A consistent transition to imaginary frequencies requires the both branches of the spectrum are to be taken into account. The contribution to the Casimir energy due to the surface modes and photonic modes can be separated only in terms of real frequencies. Upon transition to imaginary frequencies these contributions are indivisible. Presented consideration justifies rigorously the imaginary frequency representation for the Casimir energy of a compact infinite cylinder that has been used in many previous papers dealing with investigation of this energy.

It is worth noting that the mathematical consideration presented here is completely applicable to the Lifshitz configuration, namely, to an infinite dielectric plate placed in vacuum (dielectric films).

\section{Acknowledgments}

This study has been accomplished by the financial support of Russian Foundation for Basic Research (Grant 06-01-00120) and the Heisenberg-Landau Program.

\section{References}

[1] Lifshitz E M and Pitaevskii L P 1980 Landau and Lifshitz Course of Theoretical Physics: Statistical Physics Part 2 (Washington: Butterworth-Heinemann)

[2] Kittel Ch 1995 Introduction to Solid State Physics, 7th edn (New York: Wiley)

[3] Ford L H 1998 Phys. Rev. D 38528

[4] Ford L H 1993 Phys. Rev. A 482962

[5] Henkel C, Joulain K, Mulet J-Ph and Greffet J-J 2004 Phys. Rev. A 69023808

[6] Genet C, Intravaia F, Lambrecht A and Reynaud S 2004 Ann. Fond. L. de Broglie 29311 (Preprint quant-ph/0302072)

[7] Intravaia F and Lambrecht A 2005 Phys. Rev. Lett. 94110404

[8] Bordag M 2006 J. Phys. A: Math. Gen. 396173 (2006)

[9] Lifshitz E M 1965 Zh. Eksp. Teor. Fiz. 2994 [English transl.: Soviet Phys. JETP 273 (1956)]

[10] Dzyaloshinskii I E, Lifshitz E M and Pitaevskii L P 1959 Zh. Eksp. Teor. Fiz. 37229 [English transl.: Soviet Phys. JETP 10161 (1960)]

[11] Dzyaloshinskii I E, Lifshitz E M and Pitaevskii L P 1961 Adv. Phys. 10 165; Usp. Fiz. Nauk 73 381 (1961) [English transl.: Soviet Phys. Usp. 4153 (1961)] 
[12] Spruch L and Tikochinsky Y 1993 Phys. Rev. A 484213

[13] Tikochinsky Y and Spruch L 1993 Phys. Rev. A 484223

[14] van Kampen N G, Nijboer B R A and Schram K 1968 Phys. Lett. A 26307

[15] Ninham B W, Parsegian V A and Weiss G H 1970 J. Stat. Phys. 2323

[16] Gerlach E 1971 Phys. Rev. B 4393

[17] Schram K 1973 Phys. Lett. A 43282

[18] Langbein D 1973 Solid State Commun. 12853

[19] Klimchitskaya G L, Mohideen U and Mostepanenko V M 2000 Phys. Rev. A 61062107

[20] Nesterenko V V, Lambiase G and Scarpetta G 2004 Riv. Nuovo Cimento 27 No 6 1-74 (Preprint hep-th/0503100)

[21] DeRaad (Jr.)L L and Milton K 1981 Ann. Phys., NY 136229

[22] Brevik I and Nyland G H 1994 Ann. Phys., NY 230321

[23] DeRaad (Jr.) L L 1985 Fortschr. Phys. 33117

[24] Milton KA, Nesterenko A V and Nesterenko V V 1999 Phys. Rev. D 59105009 (Preprint hep-th/9711168v3)

[25] Lambiase G, Nesterenko V V and Bordag M 1999 J. Math. Phys. 406254 (1Preprint hep-th/9812059)

[26] Nesterenko V V and Pirozhenko I G 2000 J. Math. Phys. 414521 (Preprint hep-th/9910097)

[27] Nesterenko V V and Pirozhenko I G 1999 Phys. Rev. D 60125007 (Preprint hep-th/9907192)

[28] Bordag M and Pirozhenko I G 2001 Phys. Rev. D 64025019

[29] Cavero-Peláez I and Milton K A 2005 Ann. Phys., NY 320108 (2005) (Preprint hep-th/0412135v2)

[30] Cavero-Peláez I and Milton K A 2006 J. Phys. A: Math. Gen. 396225 (Preprint hep-th0511171)

[31] Cavero-Peláez I and Milton K A 2007 J. Phys. A: Math. Gen. 403607 (Preprint hep-th0607154v2)

[32] Barton G 2001 J. Phys. A: Math. Gen. 344083

[33] Gosdzinsky P and Romeo A 1998 Phys. Lett. B 441265 (Preprint hep-th/9809199)

[34] Klich I and Romeo A 2000 Phys. Lett. B 476369 (Preprint hep-th/9912223)

[35] Romeo A and Milton K A 2005 Phys. Lett. B 621 309(Preprint hep-th/0504207)

[36] Romeo A and Milton K A 2006 J. Phys. A: Math. Gen. 396703

[37] Brevik I and Romeo A 2006 Evaluation of the Casimir Force for a Dielectric-diamagnetic Cylinder with Light Velocity Conservation Condition and the Analogue of Sellmeir's Dispersion Law (Preprint hep-th/0601211)

[38] Schaden M 2006 Semiclassical Electromagnetic Casimir Self-Energies (Preprint hep-th/0604119)

[39] Nesterenko V V 2006 J. Phys. A: Math. Gen. 396609 (Preprint hep-th/0511018)

[40] Stratton J A 1941 Electromagnetic Theory (New York: McGraw-Hill)

[41] Whittaker E T 1904 Proc. London Math. Soc. 1367

[42] Nisbet A 1955 Proc. Roy. Soc. London Ser. A 231250

[43] Gradshteyn I S and Ryzhik I M 2000 Table of Integrals, Series, and Products 6th edn (New York: Academic Press)

[44] Lord Rayleigh 1897 Phil. Mag. and J. Science $\mathbf{4 3} 125$

[45] Hondros D 1909 Ann. der Phys. 30905

[46] Hondros D 1909 Phys. Z. 10804

[47] Hondros D and Debye P 1910 Ann. der Phys. 32 No 8465

[48] Schelkunoff S A 1943 Electromagnetic waves (New York: D. Van Nostrand Co.)

[49] Katzenelenbaum B S 1949 Zh. Techn. Fiz. 19 1168; 1182 [in Russian]

[50] Borgnis E and Papas C H 1958 Electromagnetic waveguides and Resonators In Encyclopedia of Physics, Ed. S. Flüge; vol XVI (Berlin: Springer) pp. 285-422

[51] Snitzer E 1961 J. Opt. Soc. Am. 51 No 5491

[52] Marcuse D 1974 Theory of dielectric optical waveguides (New York: Academic Press)

[53] Newton R G 2002 Scattering Theory of Waves and Particles (Dover, New York)

[54] Landau L D and Lifshitz E M 1996 Statistical Physics Part 1, 3rd edn (Oxford: ButterworthHeinemann) 